\title{
AVALIAÇÃO DA ALIMENTAÇÃO ESCOLAR E DO ESTADO NUTRICIONAL DE ADOLESCENTES
}

\author{
EVALUATION OF SCHOOL DIET AND NUTRITIONAL STATE AMONG TEENAGERS
}

\author{
Thiécla Katiane Rosales Silva $a^{a^{*}}$, Gabriela Antunes Camargo ${ }^{b^{*}}$, \\ Carla Evelyn Coimbra Nuñez ${ }^{\mathrm{c}^{* *}}$, Catiuscie Cabreira Silva ${ }^{\mathrm{d}^{*}}$, Cintia Reis Ballard ${ }^{* *}$ \\ athieclarosales@gmail.com, 'bantunescamargo@gmail.com, 'ccarlaecn@yahoo.com.br, 'cathicabreira@hotmail.com, \\ ecintia.reis.ballard@gmail.com \\ *Universidade Estadual do Centro-Oeste - Guarapuava (PR), Brasil \\ **Universidade Estadual de Campinas - Campinas (SP), Brasil
}

Data de recebimento do artigo: 22/09/2016

Data de aceite do artigo: 15/02/2017

\section{RESUMO}

Introduçáo e objetivo: $\mathrm{O}$ estudo objetivou avaliar a composição nutricional da merenda escolar em um colégio da rede estadual de ensino da regiāo Centro-Oeste do Paraná. Materiais e métodos: A avaliação foi realizada por meio da análise dos rótulos dos alimentos utilizados no preparo da merenda escolar, considerando-se a quantificação de calorias totais, gorduras totais, gorduras saturadas e gorduras trans, sendo esses valores comparados com o Programa Nacional de Alimentaçáo Escolar. Adicionalmente, realizouse a avaliação do estado nutricional de estudantes adolescentes matriculados no período vespertino, por intermédio de técnicas antropométricas, considerando: sexo, idade, peso, estatura e índice de massa corporal. Resultados e discussão: Foram recrutados 154 escolares de ambos os sexos, com média de idade de 12,5 anos $(\mathrm{dp} \pm 1,16)$. Entre estes, $66,88 \%$ encontravam-se eutróficos e $31,17 \%$ estavam com excesso de peso. As médias de consumo diário por aluno de calorias totais, gorduras totais, gorduras saturadas e gorduras trans, foram de: $355,30 \mathrm{kcal} / \mathrm{dia}, 1,28 \%, 1,25 \%$ e $0,22 \%$, respectivamente. Somente a quantidade calórica estava compatível com os valores de referência da legislação, representando $101,51 \%$ de adequação. Conclusão: $\mathrm{O}$ excesso de peso observado pode ter sido favorecido pela existência de cantina na escola e hábitos alimentares inadequados. Sendo assim, esses dados apontam que a alimentaçáo escolar deve ser adequada e suprir as necessidades nutricionais, a fim de proporcionar aos estudantes um bom rendimento escolar e que o nutricionista possa atuar adequando a composiçáo da merenda que lhes é oferecida, contribuindo beneficamente com açóes de promoção à saúde, bem-estar e desenvolvimento dos adolescentes.

Palavras-chave: Ácidos graxos trans; adolescentes; alimentação escolar; gorduras; transição nutricional.

\section{ABSTRACT}

Introduction and objective: The study aimed to evaluate the nutritional composition of the meals served in a state school in the Midwest region of Paraná, Brazil. Materials and methods: The evaluation was performed by analyzing the labels of the food used to prepare the meals, considering the quantification of total calories, total fat, saturated fat and trans fats, and comparing these values with the National School Feeding Programme (PNAE). Moreover, this study assessed the nutritional status of schoolchildren enrolled in the afternoon period by using anthropometric techniques, considering the following data: gender, age, weight, height, and body mass index. Results and discussion: In total, 154 adolescents of both genders were evaluated, with a mean age of 12.5 years $(\mathrm{SD} \pm 1.16)$. Among these, $66.88 \%$ were eutrophic and $31.17 \%$ were overweight (overweight and obesity). The average daily consumption per student relative to total calories, total fat, saturated fat and trans fats were: 355.30 $\mathrm{kcal} / \mathrm{day}, 1.28 \%, 1.25 \%$, and $0.22 \%$, respectively. Only caloric intake was adequate with the reference values of legislation, representing $101.51 \%$ of adequacy. Conclusion: Overweight observed may have been favored by the 
existence of the school canteen and by poor eating habits. Thus, these data indicate both that school meals must be adequate and supply the nutritional needs in order to provide to students a good school performance as well as the importance of a professional nutritionist within schools, adjusting the composition of school lunches and contributing beneficially with actions to promote health, welfare and development of adolescents.

Keywords: Trans fatty acids; adolescents; school feeding; fats; nutritional transition.

\section{Introdução}

A alimentação escolar pode ser caracterizada como todo alimento que o aluno recebe no ambiente escolar durante o período letivo, objetivando oferecer aporte energético e nutricional que proporcione adequado crescimento e desenvolvimento do alunato. A alimentação no âmbito escolar subsidia o rendimento escolar, a aprendizagem e implica na formação de hábitos alimentares dos estudantes 1 . Salienta-se também, que é por meio da oferta da alimentação escolar, das ações de educaçáo alimentar e nutricional que se favorece um estado de saúde considerado apropriado ${ }^{2,3}$. Nesse contexto a escola pode ser considerada um excelente local para realizar avaliaçóes antropométricas com o intuito de conhecer o perfil nutricional de estudantes adolescentes ${ }^{4,5}$.

$\mathrm{Na}$ busca de atender os objetivos da alimentaçáo escolar, foi implantado no Brasil em 1955 o Programa Nacional de Alimentaçáo Escolar (PNAE), esse programa atende alunos de toda a educaçáo básica do ensino público no país. O PNAE determina que os cardápios oferecidos aos escolares sejam elaborados levando em consideração o perfil epidemiológico da população atendida e que sejam utilizados alimentos básicos da tradição local e da diversidade agrícola da região. Além disso, a refeição oferecida deve suprir $15 \%$ da necessidade diária do aluno, o que equivale a 350 quilocalorias (kcal), 15 a $30 \%$ de gorduras totais, $10 \%$ de gorduras saturadas e $1 \%$ de ácidos graxos trans $s^{2,3}$.

A adoção de hábitos alimentares saudáveis na adolescência pode evitar o aparecimento de problemas de saúde na vida adulta ${ }^{6}$, porém, estudos apontam que os jovens estão consumindo cada vez mais alimentos com quantidades elevadas de calorias ${ }^{7}$ e industrializados, que contêm teores elevados de gorduras saturadas e gorduras trans ${ }^{8}$. O excesso dessas gorduras na dieta pode estar associado a algumas doenças, como as cardiovasculares e obesidade e e também outras doenças crônicas não transmissíveis ${ }^{10}$. Ressalta-se que o consumo excessivo de lipídeos na forma de gorduras saturadas é o principal fator determinante na elevação das concentrações plasmáticas de colesterol low density lipoproteins (LDL), podendo contribuir para o desenvolvimento de dislipidemias ${ }^{11}$.

Segundo o Ministério da Saúde ${ }^{12}$, a obesidade está crescendo entre os brasileiros e atinge cerca de 10,9\% dos jovens de 12 a 17 anos. Sabe-se que algumas ações dos jovens contribuem para essa estatística e estão associadas com o ganho de peso e obesidade. Já está comprovado que a alimentação dos adolescentes tem sido constituída por alimentos pouco saudáveis, como salgadinhos industrializados, refrigerantes, sucos artificiais, doces em geral, comidas de preparação rápida e reduzida ingestão de hortaliças e frutas, assim como a prática de dietas monótonas e modismos alimentares, demonstrado pela substituição de algumas refeições diárias ${ }^{10,13-15}$.

Tendo em vista os pontos negativos sobre o consumo de gorduras trans, Hissanaga ${ }^{16}$ desenvolveu em 2009 um método para controlar a utilização de gordura trans - CGTR em unidades produtoras de refeiçóes, por meio da monitorização de pontos críticos de controle (HACCP) e sistema de avaliação da qualidade nutricional e sensorial (AQNS) de ácidos graxos trans na produçáo de refeiçóes. O CGTR visa contribuir para a oferta de refeiçóes mais saudáveis sob o ponto de vista da qualidade nutricional ${ }^{16}$.

Diante disso, o presente estudo destinou-se a identificar a composiçáa dos alimentos fornecidos pela merenda escolar, quantificando os valores de calorias totais, gorduras totais, gorduras saturadas e gorduras trans, bem como verificar o estado nutricional dos alunos.

\section{Métodos}

Pesquisa de caráter quantitativo longitudinal, realizada em um colégio da rede estadual de ensino da regiăo centro-oeste do Paraná por um período de 30 dias letivos. A refeição avaliada foi a merenda do período vespertino. Para verificar as quantidades de calorias totais, gorduras totais, gorduras saturadas e gorduras trans, anotou-se inicialmente os componentes e preparaçóes servidos pela merenda escolar do local e observou-se os rótulos dos alimentos utilizados no preparo das refeiçóes.

Para detectar a presença de gorduras trans, levou-se em consideração alguns preceitos do método CGTR (Anexo 1) desenvolvido por Hissanaga ${ }^{16}$, observando nos rótulos dos ingredientes a presença ou não das seguintes nomenclaturas: gorduras trans, ácidos graxos trans e gordura vegetal hidrogenada. Como no cardápio da escola não havia presença de frituras por imersão que poderiam atingir temperaturas superiores a $180^{\circ} \mathrm{C}$, 
o que pode contribuir para a formação desse tipo de gordura durante o processo de cocção, os outros itens do método CGTR não foram utilizados e nem aplicados.

Posteriormente, para a quantificação final dos itens avaliados, foram pesadas as quantidades diárias de alimentos produzidos com ajuda da balança eletrônica modelo 3400 Toledo com capacidade de até $30 \mathrm{~kg}$ e precisão de $0,05 \mathrm{~g}$. Realizou-se a contagem do número de alunos que se alimentaram da merenda escolar e descontados os valores médios de sobras e restos. Por fim, compararam-se, os números encontrados com os valores de referência do PNAE.

Para encontrar a média total dos itens avaliados, foram pesados o desperdício, levando em consideração as sobras (alimentos produzidos e não foram servidos) e os restos (alimentos que foram servidos, porém, descartados pelos alunos) por um período de amostragem de 4 dias.

Em relação aos aspectos éticos da pesquisa, o estudo foi previamente avaliado e aprovado pelo Comitê de Ética em Pesquisa (Comep) da Universidade Estadual do Centro-Oeste (Unicentro) sob o parecer de número 684.326 (Anexo 2). Ressalta-se que os nomes dos alunos foram mantidos em sigilo, sendo usadas somente as iniciais para identificá-los.

Foram recrutados 154 estudantes adolescentes de ambos os sexos, com idade entre 10 a 15 anos, matriculados no período da tarde, pertencentes do $6^{\circ}$ ao $9^{\circ}$ ano de ensino, que aceitaram participar da pesquisa e assinaram o termo de assentimento, além disso, os responsáveis legais deveriam concordar com a participação dos escolares no estudo e assinar o termo de consentimento livre e esclarecido.

A avaliação nutricional foi realizada por um único pesquisador, treinado, para que a validade dos dados antropométricos não fosse comprometida. Foram considerados: peso, estatura e índice de massa corporal (IMC). Para o registro de peso e estatura, utilizou-se balança eletrônica portátil G-TECH, com capacidade de $150 \mathrm{~kg}$, divisão de 100 gramas e fita métrica inelástica fixada na parede. A classificação foi realizada segundo World Health Organization (WHO) 17,18, seguindo os pontos de corte de IMC por idade estabelecidos para adolescentes de 10 a 19 anos, os quais consideram: <percentil 3=baixo IMC para idade; $\geq$ percentil 3 e <percentil 85=IMC adequado; $\geq$ percentil 85 e $<$ percentil $97=$ sobrepeso e $\geq$ percentil $97=$ obesidade. Outros dados também foram ponderados, como: idade, sexo e série pertencente. Junto à antropometria os alunos respondiam um questionário (Apêndice 3) sobre a frequência de consumo do lanche da escola, onde podiam assinalar as seguintes opçóes: 1 vez por semana; 2 vezes por semana; 3 vezes por semana; 4 vezes por semana e todos os dias. Quando o aluno relatava nunca se alimentar da merenda disponibilizada pelo colégio, anotava-se o motivo que o levava a náo consumi-la.

Todos os dados coletados foram tabulados no programa Microsoft Excel, 2010. Foram realizadas as análises estatísticas descritivas e a correlação de Pearson por meio do programa estatístico Statistical Analysis System (SAS), versão 9.2.

\section{Resultados}

A amostra final foi composta por 154 adolescentes, sendo $63,64 \%$ do sexo feminino. As meninas apresentaram idade média de 11,8 anos $(\mathrm{dp} \pm 1,13)$ e os meninos 12 anos $(\mathrm{dp} \pm 1,21)$. Os valores médios por aluno obtidos referentes a calorias, gorduras totais, gorduras saturadas, gorduras trans e os percentuais que representaram os valores da adequaçáo do PNAE estáo descritos na tabela 1 , o valor resultante do desperdício foi 35\%.

Tabela 1: Valores médios de calorias totais, gorduras totais, gorduras saturadas e gorduras trans por aluno e adequação em relação aos valores de referência do PNAE.

\begin{tabular}{lcccc} 
& Valores obtidos* & $\begin{array}{c}\text { \% em relaçáo ao } \\
\text { PNAE** }\end{array}$ & $\begin{array}{c}\text { Valores de adequaçáo } \\
\text { PNAE }\end{array}$ & $\begin{array}{c}\text { Adequaçáo*** } \\
\text { Calorias totais }(\text { Kcal) }\end{array}$ \\
Gorduras totais & 355,30 & 101,51 & 350 & Adequado \\
\hline Gorduras Saturadas & $9,54 \mathrm{~g}$ & 1,28 & 15 a $30 \%$ & Inadequado \\
Gorduras Trans & $5,67 \mathrm{~g}$ & 1,25 & $10 \%$ & Inadequado \\
\hline
\end{tabular}

*Valores médios obtidos por 30 dias através da análise dos rótulos dos ingredientes, suas quantidades, pesagem diária de alimentos produzidos, contagem do número de alunos que pegavam o lanche, desconto dos valores médios de desperdício.

**Comparação com os percentuais de referência do PNAE, o qual destaca que a refeição oferecida deve suprir 15\% da necessidade diária do aluno, o que equivale a 350 quilocalorias (kcal), 15 a 30\% de gorduras totais, $10 \%$ de gorduras saturadas e $1 \%$ de gorduras trans.

***Adequação: Somente os valores de calorias totais e gorduras trans encontravam-se adequados. Gorduras trans foram consideradas adequadas pelo fato da legislaçắo nấo ter estabelecido um valor mínimo de recomendação. 
A Tabela 2 apresenta os valores obtidos na avaliação antropométrica.
A Tabela 3 demonstra a frequência de consumo do lanche durante a semana entre os escolares.

Tabela 2: Classificação do Estado Nutricional de adolescentes de 10 a 15 anos, segundo o sexo de um colégio da rede estadual de ensino de Guarapuava (PR), 2014.

\begin{tabular}{lcccccccccc} 
EN & \multicolumn{2}{c}{ Baixo peso } & \multicolumn{2}{c}{ Eutrofia } & \multicolumn{2}{c}{ Sobrepeso } & \multicolumn{2}{c}{ Obesidade } & \multicolumn{2}{c}{ Total } \\
Sexo & $\mathbf{N}$ & $\mathbf{0}$ & $\mathbf{N}$ & $\mathbf{\%}$ & $\mathbf{N}$ & $\mathbf{\%}$ & $\mathbf{N}$ & $\%$ & $\mathbf{N}$ & $\%$ \\
Feminino & 3 & 3,06 & 67 & 68,36 & 9 & 9,18 & 19 & 19,38 & 98 & 63,64 \\
Masculino & 0 & 0 & 36 & 64,28 & 13 & 23,21 & 7 & 12,5 & 56 & 36,36 \\
\hline Ambos & 3 & 1,95 & 103 & 66,88 & 22 & 17,53 & 26 & 13,64 & 154 & 100 \\
\hline
\end{tabular}

\%: porcentagem de valores médios; EN: estado nutricional ( $\mathrm{dp} \pm 0,74) ; \mathrm{N}$ : número; Total: número e porcentagem total de alunos distintos por sexo. Classificação do estado nutricional segundo a $\mathrm{WHO}^{17,18}$.

Tabela 3: Frequência de consumo da merenda escolar

\begin{tabular}{lcccccc} 
Frequência & Geral $(\mathbf{N})$ & $(\%)$ & Geral $(\mathbf{N})$ & (\%) & Geral (N) & $(\%)$ \\
Nunca & 55 & 35,71 & 36 & 65,45 & 19 & 34,55 \\
\hline 1 vez na semana & 34 & 22,08 & 18 & 52,94 & 16 & 47,06 \\
2 vezes na semana & 26 & 16,88 & 17 & 65,38 & 9 & 34,62 \\
\hline 3 vezes na semana & 14 & 9,09 & 11 & 78,57 & 3 & 21,43 \\
\hline 4 vezes na semana & 5 & 3,25 & 2 & 40 & 3 & 60 \\
\hline 5 vezes na semana & 20 & 12,99 & 14 & 70 & 6 & 30 \\
Total & 154 & 100 & 98 & 63,64 & 56 & 36,36
\end{tabular}

Valores médios obtidos através do questionário de frequência do consumo do lanche na escola, onde podiam assinalar as seguintes opçóes: 1 vez por semana; 2 vezes por semana; 3 vezes por semana; 4 vezes por semana e todos os dias. N: número; \%: porcentagem; Geral: total de alunos recrutados, somando os do sexo feminino e masculino.

Ao serem questionados em relação à frequência de consumo da merenda escolar, 55 alunos não se identificaram com nenhuma das alternativas contidas no questionário, visto que nunca consumiam a merenda escolar. Como no questionário não havia a opção "nunca", foram anotados os motivos pelos quais eles não consumiam, sendo eles: A compra de lanche na cantina $(41,97 \%)$ e a falta de fome no horário em que o lanche é servido na escola (41,07\%). Alguns alunos relataram náo consumir a merenda porque traziam comida de fora da escola $(8,93 \%)$ e outros pelo fato de não gostarem da merenda $(8,93 \%)$. Adicionalmente, ao correlacionar as variáveis: ingestão da merenda escolar e o estado nutricional, não se observou correlação estatis-

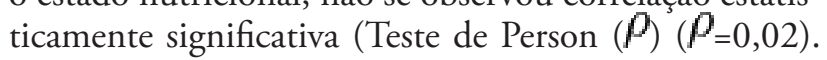
Para que o valor do coeficiente de correlação de Pearson fosse perfeitamente significativo no estudo, este deveria apresentar valores de 1 ou -1 .

A escola apresenta um cardápio próprio, elaborado de acordo com a disponibilidade de alimentos provenientes do Estado, produtos perecíveis e prazo de validade. As refeiçóes servidas se caracterizam por um lanche salgado e mais calórico no início e no fim da semana e preparaçóes geralmente doces no meio da semana. Os alimentos que faziam parte do cardápio podem ser visualizados na Tabela 4 .

Tabela 4: Componentes e preparações principais da merenda escolar

\begin{tabular}{llll} 
Preparaçóes Doces & Preparaçóes salgadas & Frutas e hortaliças & Bebidas \\
Arroz doce & Alho triturado* & Alface & Achocolatado \\
Barra de cereal & Arroz parboilizado & Agriáo & Bebida láctea \\
Barra de goiaba & Batata inglesa cozida & Banana & Composto de café com leite \\
Biscoito de castanha-do-Pará & Cachorro quente & Beterraba & Composto de coco com \\
Biscoito de maizena & Canja & Brócolis & Leite \\
Bolacha caseira & Carnes bovina/frango/suína & Cenoura & Suco de maçã \\
\hline
\end{tabular}


Tabela 4: Continuação

\begin{tabular}{|c|c|c|c|}
\hline Preparaçóes Doces & Preparaçóes salgadas & Frutas e hortaliças & Bebidas \\
\hline Canjica & Cebola* & Couve & Suco de uva integral \\
\hline Cuca de goiaba & Ervilha* & Pepino & \\
\hline Rosquinha de baunilha & Farofa de milho & Repolho & \\
\hline $\begin{array}{l}\text { Pão caseiro com doce de uva/figo } \\
\text { ou margarina }\end{array}$ & Feijão preto & Rúcula & \\
\hline Rosquinha de chocolate & Macarrāo com molho & Tangerina & \\
\hline Rosquinha de leite & Milho & & \\
\hline \multirow[t]{4}{*}{ Sagu } & Óleo de soja* & & \\
\hline & Polenta com carne moída & & \\
\hline & Purê de batata formulado & & \\
\hline & Virado de feijão & & \\
\hline
\end{tabular}

Alimentos ou preparaçôes constituintes do cardápio da merenda escolar e distribuídos por doces, salgados, frutas/hortaliças e bebidas. *Componentes de algumas preparaçōes salgadas.

\section{Discussão}

Observou-se que $35,71 \%$ dos alunos nunca consumiam a merenda escolar, representando o maior percentual relatado em relação ao consumo da merenda entre os adolescentes, sendo que o PNAE visa atender todos os alunos da rede pública de ensino do país. Uma possível explicaçáo poderia ser a preferência por alimentos disponíveis em cantinas particulares durante a permanência no intervalo entre as aulas, conforme observado por Leme et al. ${ }^{19} \mathrm{em}$ uma pesquisa transversal feita com 83 adolescentes de ambos os sexos na cidade de São Paulo. Em uma escola da rede pública de São Luís do Maranhão, Carvalho et al..$^{20}$, também obtiveram resultados alarmantes, os quais comprovaram que de 100 alunos adolescentes de 15 a 17 anos, 51,00\% adquiriam o lanche na cantina, e 12,00\% levavam de casa ${ }^{21}$.

Considerando a variável de IMC, a maioria dos adolescentes apresentou diagnóstico nutricional dentro da faixa de eutrofia, representando 66,88\%. Esses dados corroboram com os achados de Bertin et al. ${ }^{21}$, onde em um estudo descritivo de corte transversal, realizado com 1610 alunos de 12 anos a 16 anos de idade matriculados na rede pública de ensino da cidade de São Mateus do Sul, situada na região Sul do Paraná, constataram que a maioria dos adolescentes apresentava estado nutricional eutrófico, sendo representado por um percentual de $84,10 \%$.

$\mathrm{O}$ esperado era que a merenda oferecida pela escola em questáo estivesse adequada em todos os itens avaliados, colaborando para a manutenção de um peso saudável. Ao invés disso, o que se encontrou foram uma inadequação em teor de gorduras e uma média de consumo calórico por aluno de $355,30 \mathrm{kcal} / \mathrm{dia}$, variando entre barra de cereal composto por $84,81 \mathrm{kcal}$ a virado de feijão, carne suína e couve com $614,0 \mathrm{kcal}$. Embora a média de calorias totais esteja compatível com o referencial do PNAE, os valores mostram uma diferença entre o oferecimento de calorias durante os dias avaliados, visto que em determinados dias consumiam um valor calórico muito baixo e em outros valores calóricos muito altos.

Todos os rótulos analisados declaravam somente os valores de gorduras totais, gorduras saturadas e gorduras trans, não aparecendo em nenhum deles valores referentes a gorduras poli-insaturadas e monoinsaturadas. Os valores abaixo do limite inferior de gorduras totais, gorduras saturadas e gorduras trans podem ser explicados pela preocupação do Estado e da escola em atender o compromisso do PNAE, buscando oferecer aos alunos alimentos produzidos de forma sustentável, priorizando os produtos da regiāo, favorecendo os alimentos orgânicos da agricultura familiar, portanto, mais saudáveis e pobres em gorduras. Como algumas pesquisas apontam a diminuição do consumo de alimentos tradicionais como o arroz, feijão, frutas e hortaliças, e o aumento no consumo de produtos industrializados, ricos em açúcar e em gorduras totais e gorduras saturadas, fato que modifica o estado nutricional da população, dessa forma, evidencia-se o papel do Estado na construçấo e consolidaçáo de modelos alimentares que possibilitem o enfrentamento dos problemas relacionados à produção e de consumo através de uma boa alimentação escolar ${ }^{22-25}$. Em 2013, o governo do Paraná aumentou os investimentos para compra de alimentos da agricultura familiar para a merenda. Existem 83 itens alimentícios que podem ser obtidos, destacando-se: carne, ovos, feijão, frutas, hortaliças, iogurte e similares, legumes, leite e panificados, além disso, em um período de três anos, a quantidade de alimentos orgânicos na merenda dos alunos apresentou um aumento significativo, demonstrando que a agricultura familiar tem se tornado prioridade nas escolas do Paraná, visto que esse tipo de alimento ajuda a melhorar o aporte de nutrientes, incentiva hábitos alimentares mais saudáveis e estimula a economia dos municípios ${ }^{26}$.

$\mathrm{O}$ excesso de peso foi observado em $31,17 \%$ dos casos, apontando que $17,53 \%$ dos alunos estavam com sobrepeso e $13,64 \%$ com obesidade. Sendo a obesidade mais prevalente no sexo feminino: $19,38 \%$ e $12,5 \%$ no 
sexo masculino. Já a classificação de baixo peso só foi vista em 3,06\% no sexo feminino. Tais números indicam que esse grupo de adolescentes estudados passa por um período denominado transiçáo nutricional, processo pelo qual há alteraçôes nos padrôes dietéticos e nutricionais, onde é visível a diminuição do baixo peso e a prevalência do aumento do sobrepeso e da obesidade ${ }^{27,28}$. Em um estudo transversal realizado por Dantas et al., $2010^{29}$, com uma amostra de 134 adolescentes matriculados em uma escola privada na cidade de Santa Inês, no Maranhão, foi possível verificar que $38,8 \%$ dos alunos encontravam-se acima do peso, o que demonstra que o excesso de peso não está presente somente em escolas públicas da região Sul do país, mas também em escolas particulares e em demais regiōes do país, como o Nordeste. Segundo pesquisas realizadas pelo Instituto Brasileiro de Geografia e Estatística (IBGE) e Pesquisa de Orçamentos Familiares (POF) nos anos de 1974-1975, 1989, 2002-2003 e 2008-2009 com $\mathrm{o}$ intuito de analisar o estado nutricional de adolescentes de 10 a 19 anos, baseado nas medidas antropométricas, ficou constatado ao longo dos quatro inquéritos que a tendência secular entre os jovens de todas as regióes do Brasil é o declínio da prevalência de déficit de peso e a prevalência de excesso de peso aumentando continuamente. Nos 34 anos decorridos de 1974-1975 a 2008-2009, a prevalência de excesso de peso aumentou em seis vezes no sexo masculino (de 3,7\% para 21,7\%) e em quase três vezes no sexo feminino (de 7,6\% para $19,4 \%)^{13}$.

Em uma pesquisa de caráter transversal realizada em Sorocaba - São Paulo, também com 154 adolescentes escolares da rede pública de ensino, Souza et al., 20137, identificaram que $3,2 \%$ adolescentes estavam com baixo peso, resultados que se assemelham aos achados do nosso estudo. Foi possível observar que o baixo peso é mais presente nos alunos que pertencem a um nível socioeconômico menor e que se alimentam da merenda escolar todos os dias e, que certamente a merenda tem uma importante contribuição na alimentação diária desse aluno, sendo em muitos casos a principal fonte de alimentação. Esse fato reforça a ideia de que o PNAE é essencial para garantir a segurança alimentar dos alunos de todas as classes sociais, inclusive os mais desfavorecidos financeiramente ${ }^{30}$. A alimentação escolar tem potencial para favorecer um bom estado nutricional aos escolares, desde que as refeiçóes sejam elaboradas e oferecidas em quantidade e qualidade adequadas ${ }^{31}$.

Uma limitação do estudo é o fato de não ter sido possível a realização da pesagem do desperdício da merenda durante todos os dias de avaliação. Outra limitação é que a pesquisa não foi realizada com todos os alunos da instituição. Porém, apesar das limitações, essa investigação é inédita na região e os resultados obtidos apresentam um pouco da realidade da merenda escolar e do estado nutricional dos estudantes dos colégios estaduais do Paraná.

\section{Conclusão}

Este estudo proporcionou uma análise quantitativa da alimentaçáo escolar. Os resultados encontrados indicaram que mesmo as distribuiçóes de gorduras totais e gorduras saturadas na merenda estando abaixo das recomendaçóes do PNAE, grande parte dos adolescentes apresentam sobrepeso e obesidade. Isso pode ser explicado por escolhas inadequadas na alimentaçáo, que náo estão incluídas na merenda escolar.

Como não houve correlaçâo entre o estado nutricional e a frequência do consumo da merenda escolar, a existência de cantina na escola pode indicar que há um favorecimento do consumo de alimentos ricos em açúcares e gorduras, culminando assim no excesso de peso. Além disso, os hábitos alimentares inadequados vivenciados em casa pelas famílias podem contribuir para esse agravante.

Destaca-se a relevância em realizar a verificação dos produtos que são vendidos na cantina e a monitorização constante do estado nutricional dos jovens avaliados por meio das mesmas técnicas antropométricas empregadas nessa pesquisa. Ressalta-se ainda que a identificação do estado nutricional possa subsidiar intervençóes nutricionais adequadas para essa população.

Os dados obtidos apontam que a alimentação escolar deve ser adequada e suprir as necessidades nutricionais, a fim de proporcionar aos estudantes um bom rendimento escolar e que o nutricionista possa atuar adequando a composição da merenda que lhes é oferecida, favorecendo os alunos que apresentam excesso de peso e déficit nutricional, e contribuir beneficamente com ações de promoção à saúde, bem-estar e desenvolvimento dos adolescentes. Além do mais, indicam que o colégio necessita de melhorias e ajustes na qualidade da alimentação ofertada aos escolares, na busca de colaborar para um bom crescimento, desenvolvimento, rendimento e saúde desses alunos.

\section{Referências}

1. Menegazzo M, Fracalossi K, Fernandes A, Medeiros NI. Avaliação qualitativa das preparaçóes do cardápio de centros de educação infantil. Rev Nutr. 2011;24(2):243-51.

2. Brasil. Ministério da Educação. Fundo Nacional de Desenvolvimento da Educação. Conselho Deliberativo. Resoluçáo no 38, de 16 de julho de 2009. Dispóe sobre o atendimento da alimentação escolar aos alunos da educação básica no Programa Nacional de Alimentação Escolar - PNAE [Internet]. Diário Oficial da União. Brasília, DF; 16 jul 2009 [citado em 2014 ago 13]. Disponível em: http://bit.ly/2nNrut5

3. Brasil. Lei no 11.947 , de 16 de junho de 2009. Dispóe sobre o atendimento da alimentação escolar e do Programa Dinheiro Direto na Escola aos alunos da educação básica. Diário Oficial da União. Brasília, DF; 17 jun 2009. 
4. Abrantes MM, Lamounier JA, Colosimo EA. Prevalência de sobrepeso e obesidade em crianças e adolescentes das regióes Sudeste e Nordeste. J Pediatr. 2002;78(4):335-40.

5. Anjos LA, Castro IRR, Engstrom EM, Azevedo AMF. Crescimento e estado nutricional em amostra probabilística de escolares no Município do Rio de Janeiro, 1999. Cad Saúde Pública. 2003;19(Supl 1):S171-9.

6. American Dietetic Association. Position of the American Dietetic Association: nutrition guidance for healthy children ages 2-11 years. J Am Diet Assoc. 2008;108(6):1038-47.

7. Souza JB, Enes CC. Influência do consumo alimentar sobre o estado nutricional de adolescentes de Sorocaba-SP. J Health Sci Inst. 2013;31(1):65-7.

8. Leal GVS, Philippi ST, Matsudo SMM, Toassa EC. Food intake and meal patterns of adolescents, São Paulo, Brazil. Rev Bras Epidemiol. 2010;13(3):457-67.

9. Costa AGV, Bressan J, Sabarense CM. Ácidos Graxos Trans: Alimentos e Efeitos na Saúde. Arch Latinoam Nutr. 2006;56(1):12-21.

10. Veiga GV, Cunha AS, Sichieri R. Trends in overweight among adolescents living in the poorest and richest regions of Brazil. Am J Public Health. 2004;94(9):1544-8.

11. Giuliano ICB, Coutinho MSSA, Freitas SFT, Pires MMS, Zunino JN, Ribeiro RQC. Lípideos séricos em crianças e adolescentes de Florianópolis, SC: Estudo Floripa saudável 2040. Arq Bras Cardiol. 2005;85(2):85-91.

12. Brasil. Ministério da Saúde. Secretaria de Atenção à Saúde. Departamento de Atenção Especializada. Coordenação da Política Nacional de Sangue e Hemoderivados. Manual de condutas básicas na doença falciforme [Internet]. Brasília, DF: Ministério da Saúde; 2006 [citado em 2017 mar 6]. Disponível em: http://bit.ly/2mKa4R7

13. Rosenheck R. Fast food consumption and increased caloric intake: a systematic review of a trajectory towards weight gain and obesity risk. Ob Reviews. 2008;9(6):535-47 .

14. Briefel RR, Crepinsek MK, Cabili C, Wilson A, Gleason PM. School food environments and practices affect dietary behaviors of U.S. public school children. J Am Diet Ass. 2009;109(2):91-107.

15. Brasil. Ministério da Saúde. Reportagens especiais [Internet]. 2014 [citado em 2014 out 16]. Disponível em: http://bit.ly/2mR6i63

16. Hissanaga VM. Desenvolvimento de um método para o controle da utilização de gordura trans no processo produtivo de refeiçóes. Florianópolis: Universidade Federal de Santa Catarina; 2009.

17. World Health Organization. Growth reference data for 5-19 years: BMI-for-age GIRLS [Internet]. Geneva: WHO; 2007 [citado em 2014 ago 25]. Disponível em: http://bit. ly/2mAodwd
18. World Health Organization. Growth reference data for 5-19 years: BMI-for-age BOYS [Internet]. Geneva: WHO; 2007 [citado em 2014 ago 25]. Disponível em: http://bit. ly/2n6jk $1 \mathrm{~K}$

19. Leme ACB, Philippi ST, Toasse EC. O que os adolescentes preferem: os alimentos da escola ou os alimentos competitivos? Saúde Soc. 2013;22(2):456-67.

20. Carvalho CA, Fonseca PCA, Oliveira FP, Coelho AA, Arruda SPM. Consumo de energia e macronutrientes no lanche escolar de adolescentes de São Luís, Maranhão, Brasil. Cad. Saúde Col. 2014;22(2):212-17.

21. Bertin LR, Karkle ENL, Ulbrich AZ, Neto AS, Bozza R, Araujo IQ, et al. Estado nutricional e consumo alimentar de adolescentes da rede pública de ensino da cidade de São Mateus do Sul, Paraná, Brasil. Rev. Bras. Saúde Matern. Infant. 2008;8(4):435-43.

22. Melão IB. Produtos sustentáveis na alimentação escolar: $\mathrm{O}$ PNAE no Paraná. Cad Ipardes. 2012;2(2):87-105.

23. Brasil. Conselho Nacional de Segurança Alimentar e Nutricional. Princípios e diretrizes de uma política de segurança alimentar e nutricional: textos de referência da II Conferência Nacional de Segurança Alimentar e Nutricional. Brasília, DF: Consea; 2004.

24. Brasil. Lei no 11.326, de 24 de julho de 2006. Estabelece as diretrizes para a formulação da Política Nacional da Agricultura Familiar e Empreendimentos Familiares Rurais [Internet]. Diário Oficial da Uniāo. Brasília, DF; 24 jul 2006 [citado em 2014 ago 11]. Disponível em: http://bit.ly/157rNWd

25. Triches RM, Schneider S. Alimentação escolar e agricultura familiar: reconectando o consumo à produção. Saúde Soc. 2010;19(4):933-45.

26. Paraná. Secretaria de Educação do Estado. Agricultura familiar na merenda escolar ganha destaque nacional [Internet]. 3 dez 2013 [citado em 2014 out 16]. Disponível em: http:// bit.ly/2m6pTy2

27. Batista FM, Rissin A. A transição nutricional no Brasil: tendências regionais e temporais. Cad Saúde Pública. 2003;19(1):181-91.

28. Popkin BM. The nutrition transition and obesity in the developing world. J Nutr. 2001;131(3):871-73.

29. Dantas SD, Sousa RML, Chein MBC, Brito LMO. Prevalência de sobrepeso e obesidade em adolescentes de uma escola particular em Santa Inês-MA. Rev Bras Obes Nutr Emagrec. 2010;19(4):22-30.

30. Hoffmann R. Determinantes do consumo da merenda escolar no Brasil: análise dos dados da PNAD de 2004 e 2006. Segurança Alimentar e Nutricional. 2012;19(1):33-45 .

31. Belik W, Souza LR. Algumas reflexóes sobre os programas de alimentação escolar da América Latina. Planej Polít Públicas. 2009;33(1):104-22.

\section{Como citar este artigo:}

Silva TKR, Camargo GA, Nuñez CEC, Silva CC, Ballard CR. Avaliação da alimentação escolar e do estado nutricional de adolescentes. Rev. Aten. Saúde. 2017;15(51):62-68. 